\title{
The Role of Intestinal Microbiota and Mast Cell in a Rat Model of Visceral Hypersensitivity
}

\author{
Ying-Jie Li, ${ }^{1}$ Jing Li, ${ }^{1 *}$ and Cong Dai ${ }^{2 *}$ \\ ${ }^{I}$ Department of Gastroenterology, First Affiliated Hospital, Jinzhou Medical University, Jinzhou, Liaoning, China; and ${ }^{2}$ Department of \\ Gastroenterology, First Affiliated Hospital, China Medical University, Shenyang, Liaoning; China
}

\section{Background/Aims}

To explore the role of intestinal flora and mast cells in visceral hypersensitivity (VH).

\section{Methods}

The experimental animals were divided into 4 groups: control group, VH group, VH + VSL\#3 group, and VH + ketotifen group. Stool samples were collected from each group $(n=3)$ for a further analysis using 165 ribosomal DNA gene sequence. Visceral sensitivity was evaluated by abdominal withdrawal reflex (AWR) score. Colon tissues of rats were obtained from each group. Mast cells were detected by toluidine blue staining. The degranulation of mast cells was assessed by transmission electron microscopy.

\section{Results}

$\mathrm{VH}$ rat model could successfully be induced by acetic acid enema combined with partial limb restraint method. Compared with rats in the control group, AWR score, number of mast cells, and degranulation of mast cells were increased in the VH rats, which could be reduced by administration of ketotifen or probiotic VSL\#3. Clostridium sensu stricto 1 abundance was higher in the VH group compared to the control group, which could be restored by application of probiotic VSL\#3.

\section{Conclusions}

Probiotic VSL\#3 decreases visceral sensitivity in VH rats. The mechanism may be related to mast cell and intestinal flora. Change of Clostridium sensu stricto 1 abundance may be a basis for VH observed in irritable bowel syndrome and may be prevented by specific probiotic administration.

(J Neurogastroenterol Motil 2020;26:529-538)

\section{Key Words}

Gastrointestinal microbiome; Irritable bowel syndrome; Mast cells; Probiotics; Rats

Received: January 13, 2020 Revised: May 4, 2020 Accepted: July 3, 2020

(.) This is an Open Access article distributed under the terms of the Creative Commons Attribution Non-Commercial License (http://creativecommons. org/licenses/by-nc/4.0) which permits unrestricted non-commercial use, distribution, and reproduction in any medium, provided the original work is properly cited.

${ }^{*}$ Correspondence: Jing Li and Cong Dai are equally responsible for this study.

\section{Jing Li, MD}

Department of Gastroenterology, First Affiliated Hospital, Jinzhou Medical University, No.2, section 5, Renmin Street, Guta District, Jinzhou, Liaoning 121001, China

Tel: +86-15940657929, Fax: +86-15940657929, E-mail: jz7203@139.com

Cong Dai, MD

Department of Gastroenterology, First Affiliated Hospital, China Medical University, No. 92 of Beier Road, Heping District,

Shenyang, Liaoning 110001, China

Tel: +86-18840054258, Fax: +86-18840054258, E-mail: congdai2006@126.com 


\section{Introduction}

Irritable bowel syndrome (IBS) is a clinical syndrome characterized by abdominal pain or discomfort accompanied by changes in defecation habits or frequency. The incidence of IBS is about $5.8 \%$ to $17.5 \%,{ }^{1}$ which varies due to different country and classification criteria of IBS. According to Rome IV criteria, ${ }^{2}$ IBS is classified into 4 subtypes, including diarrhea-predominant, constipationpredominant, mixed, and undetermined type. Although IBS is not life-threatening, it is characterized by a long-term course and recurrence, which leads to significant expenditure on medical resources.

The etiology of IBS is still unclear. The pathogenesis of IBS includes abnormal intestinal motility and sensation, abdominal distension, excessive neuro-hormonal stress response, and abnormal central regulatory mechanism. Visceral hypersensitivity $(\mathrm{VH})$ is the hallmark of IBS.,4 Therefore, further study of the pathogenesis of VH may reveal the mystery of IBS.

Mast cells may be involved in the pathogenesis of VH. It has been shown that mast cells can affect the gastrointestinal function. The increase of mast cell numbers can affect the intestinal mucosal barrier function, ${ }^{5}$ which leads to an increase of visceral sensitivity. ${ }^{6}$ The severity of symptoms in IBS patients was related to the number of mast cells. Stress or inflammation-induced VH could be attenuated by mast cell stabilizers. ${ }^{7}$ Mast cell deficiency eliminated TNBS-induced VH in rats. ${ }^{8}$ Mast cells release a variety of inflammatory mediators and cytokines through degranulation, including biogenic amines (eg, bradykinin and serotonin), ${ }^{9-13}$ enzymes, arachidonic acid metabolites, adenosine, neuropeptides, and cytokines under stress, which could affect adjacent nociceptive nerve fibers by activating corresponding receptors, thereby indirectly changing the sensory threshold of primary afferent neurons. At the same time, it was also found that there were Toll-like receptors on the surface of mast cells, which can recognize intestinal microorganisms and make corresponding physiological reactions. ${ }^{14-17}$

Intestinal flora plays an important role in the pathogenesis of VH. Animal and human studies have shown that bi-directional signal transduction between brain and intestine affects stress response. Intestinal flora may be an important mediator in brain-gut interaction. ${ }^{18}$ It can affect nerve pathways, nerve development and plasticity, pain perception, neurobiochemistry, and integrity of the blood-brain barrier. Epidemiology shows that the development of IBS symptoms is related to the destruction of flora in individuals. For example, IBS symptoms developed after antibiotic treatment or infection, and post-infectious IBS has a close relationship with intestinal flora. ${ }^{19}$ In addition, IBS is associated with small intestinal bacterial overgrowth. ${ }^{20}$ Clinical data showed that the intestinal flora composition in IBS patients had changed. ${ }^{18,21,22}$ Transplanting these human stools to sterile rats can lead to $\mathrm{VH}^{22}$ It was found that nonabsorbable antibiotics (bacitracin/neomycin) induced intestinal flora disorder, and reduced visceral pain-related responses in mice. ${ }^{23}$ At present, although there are many studies on intestinal flora and pathogenesis of IBS, the results are inconsistent. Hadizadeh et $\mathrm{al}^{24}$ reported that abdominal pain sensation was associated with an altered fecal microbiota composition (such as Prevotella, Blautia, Streptococcus, and Lactobacillus). But some studies found that there were no significant differences in fecal microbiota composition between IBS and general population. ${ }^{25,26}$ The relationship between intestinal flora and IBS needs further study.

Based on the above research background, the aim of this study is to explore the role of mast cells and intestinal flora in $\mathrm{VH}$ rats, further reveal the pathogenesis of $\mathrm{VH}$, and provide new therapeutic targets for IBS.

\section{Materials and Methods}

\section{Animals}

A total of 32 male specific pathogen-free Sprague-Dawley rats weighing 150-170 g were purchased from Beijing HFK bioscience Co, Ltd (Beijing, China). The rats were kept in the cleangrade laboratory animal room. The room temperature and humidity were constant. The temperature was controlled between $20^{\circ} \mathrm{C}$ and $25^{\circ} \mathrm{C}$. The food and drinking water were freely consumed by rats. At the end of the experiment, rats were euthanized to minimize the pain or discomfort. All experiments followed the guidelines of the International Council for Laboratory Animal Science (Permit No. 2018035).

\section{Experimental Protocol}

In the control group, the rats were fed under normal conditions without stimulation.

In the VH model group $(\mathrm{n}=8)$, the $\mathrm{VH}$ model was induced as follows ${ }^{27-29}$ : briefly, $1 \mathrm{~mL}$ of $4 \%$ acetic acid was injected into the colon by an enema tube through the anus (7-cm away from the anus), compressed the anus with a cotton swab for 30 seconds, injected $1 \mathrm{~mL}$ of phosphate-buffered saline into the colon, then slowly pulled out the enema tube, and fed normally for 6 days. The rats were fasted for 2 hours each every day.

In the $\mathrm{VH}+$ ketotifen group, the $\mathrm{VH}$ rats $(\mathrm{n}=8)$ were 
treated with intraperitoneal injection of ketotifen furamate $(1 \mathrm{mg} / \mathrm{kg})$ from day 7 to day $13 .{ }^{29}$ On day 14 the rats were sacrificed.

In the VH + VSL\#3 group, the VH rats $(\mathrm{n}=8)$ were treated once daily with intragastric administration of VSL\#3 from day 7 to day 13. VSL\#3 (15 mg) was dissolved in $200 \mu \mathrm{L}$ of phosphatebuffered saline. $^{29,30}$

\section{Rectal Distension Procedure}

A disposable 8-French silicone balloon-urethral catheter for pediatric was used for abdominal withdrawal reflex (AWR) in response to colorectal distention. Insertion of the catheter lubricated with paraffin oil into the rats through anus (the end of the balloon was about $7 \mathrm{~cm}$ from the anus) was performed, with fixation of the end of the catheter at $1 \mathrm{~cm}$ distal from the anus. The rats were placed on a self-made platform, after 30 minutes of adaptation, the AWR score was recorded when the distention pressure of the balloon was at 15, 30, 45, and $60 \mathrm{mmHg}$. Each expansion lasted for 30 seconds, repeated for 3 times, with an interval of 5 minutes. AWR scoring criteria: 0 : no behavioral response to balloon dilation in rats; 1 point: body immobility and head movement reduction during balloon dilation; 2 points: abdominal muscle contraction during balloon dilation, but the abdomen is not lifted off the platform surface; 3 points: abdominal muscle contraction and the abdomen lifted off the platform surface during balloon dilation; and 4 points: pelvis lifted, the body arched and the perineum left the ground during balloon dilation. ${ }^{29,31}$

Rats were sacrificed at the end of the last distension protocol. One centimeter long segments of colon $(3 \mathrm{~cm}$ and $8 \mathrm{~cm}$ from the anus, ileocecus) were removed for $\mathrm{H} \& \mathrm{E}$ staining and toluidine blue staining. ${ }^{29}$ Small pieces of colon tissue $(1 \mathrm{~mm} \times 1 \mathrm{~mm})$ were fixed in $3 \%$ phosphate buffered glutaraldehyde. It was stored in a refrigerator at $4{ }^{\circ} \mathrm{C}$ for further mast cell electron microscopic examination.

\section{Hematoxylin and Eosin Staining and Toluidine Blue Staining}

Three colon specimens were fixed in Carnoy's fixation, and 5 $\mu \mathrm{m}$ sections were processed for $\mathrm{H} \& \mathrm{E}$ staining and toluidine blue staining. ${ }^{29}$

Mast cell toluidine blue staining: paraffin-embedded section subjected to conventional dewaxing to water, stained with $0.5 \%$ toluidine blue for 10 minutes, differentiated with $0.5 \%$ glacial acetic acid, made transparent using xylene, and sealed with a neutral balsam. Under the microscope (1x71; Olympus Corporation, Tokyo, Japan), the granules of mast cells were purple-red and the nuclei were blue. The distribution of mast cells was observed under low power microscopy, and the number of mast cells was calculated for every 5 high power fields $(40 \times$ objective magnification $)$ and the mean was calculated.

\section{Electron Microscopic Examination}

Colon tissues were fixed for 2 hours in $3 \%$ phosphate buffered glutaraldehyde fixative at $4{ }^{\circ} \mathrm{C}$, subsequently bathed in a cacodylate buffer 3 times, and post-fixed for 90 minutes in a $1 \%$ buffer solution of osmium tetroxide $\left(\mathrm{OsO}_{4}\right)$. They were then dehydrated in a graded series of ethanol, immersed in propilenoxide and durcupan (SPI-Epon 812; Structure Probe, Inc, West Chester, PA, USA) and embedded in durcupan.

Stool samples were collected from rats in each group $(n=3)$. All of the stool samples were immediately stored at $-80^{\circ} \mathrm{C}$ and were transferred to Shanghai Majorbio Bio-pharm Technology Co., Ltd (Shanghai, China) where the total DNA was extracted and tested for further analysis.

\section{S Ribosomal DNA Sequencing of Fecal Microbiota}

$16 \mathrm{~S}$ ribosomal DNA (rDNA) sequencing was processed at Shanghai Majorbio Bio-pharm Technology Co., Ltd by using Illumina MiSeq System (Illumina, San Diego, CA, USA).

Microbial DNA was extracted from mouse fecal pellets using the E.Z.N.A. soil DNA Kit (Omega Bio-tek, Norcross, GA, USA) according to manufacturer's protocols. The final DNA concentration and purification were determined by NanoDrop 2000 UV-vis spectrophotometer (Thermo Scientific, Wilmington, DE, USA), and DNA quality was checked by $1 \%$ agarose gel electrophoresis. The V3-V4 hypervariable regions of the bacteria 16S rDNA gene were amplified with primers 5'-barcode-ACTCCTACGGG AGGCAGCA-3' and 806R5'-GGACTACHVGGGTWTCTA AT-3' by thermocycler polymerase chain reaction (PCR) system (GeneAmp 9700; Applied Biosystems, Union City, CA, USA). The PCR reactions were conducted using the following program: 3 minutes of denaturation at $95^{\circ} \mathrm{C}, 27$ cycles of 30 seconds at $95^{\circ} \mathrm{C}$, 30 seconds for annealing at $55^{\circ} \mathrm{C}$, and 45 seconds for elongation at $72^{\circ} \mathrm{C}$, and a final extension at $72^{\circ} \mathrm{C}$ for 10 minutes. PCR reactions were performed in triplicate $20 \mu \mathrm{L}$ mixture containing $4 \mu \mathrm{L}$ of $5 \times$ FastPfu buffer, $2 \mu \mathrm{L}$ of $2.5 \mathrm{mM}$ dNTPs, $0.8 \mu \mathrm{L}$ of each primer ( 5 $\mu \mathrm{M}), 0.4 \mu \mathrm{L}$ of FastPfu polymerase, and $10 \mathrm{ng}$ of template DNA. The resulting PCR products were extracted from a $2 \%$ agarose gel and further purified using the AxyPrep DNA Gel Extraction Kit (Axygen Biosciences, Union City, CA, USA) and quantified using QuantiFluor-ST (Promega, Medison, WI, USA) according to the 
manufacturer's protocol.

Purified amplicons were pooled in equimolar and paired-end sequenced $(2 \times 300)$ on an Illumina MiSeq platform (Illumina) according to the standard protocols by Majorbio Bio-Pharm Technology Co, Ltd (Shanghai, China).

Operational taxonomic units (OTUs) were clustered with 97\% similarity cutoff using UPARSE (version $7.1 \mathrm{http}$ ://drive5.com/ uparse/) with a novel "greedy" algorithm that performs chimera filtering and OUT clustering simultaneously. The taxonomy of each 16S rDNA gene sequence was analyzed by Ribosomal Database Project Classifier algorithm (http://rdp.cme.msu.edu/) against the Silva (SSU123) 16S rDNA database using confidence threshold of $70 \%$.

\section{Statistical Methods}

Data were expressed as mean $\pm \mathrm{SD}$. A significant difference among groups in the values (AWR score) at each distension pressure was statistically analyzed using ANOVA. A Fisher's significant difference and Student-Newman-Keuls post hoc test was

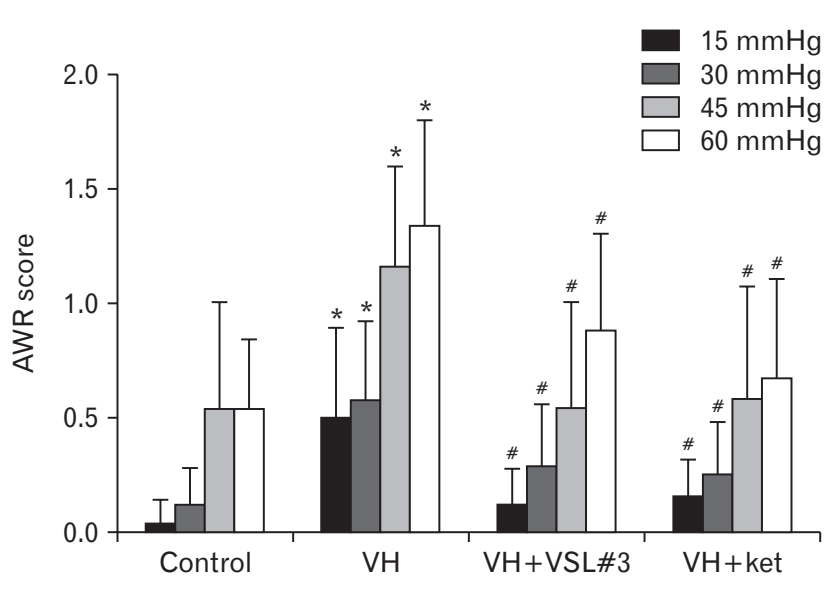

Figure 1. Comparison of abdominal withdrawal reflex (AWR) scores in different groups. AWR score in the visceral hypersensitivity $(\mathrm{VH})$ group was higher than that in the control group. After administration with ketotifen (Ket) or VSL\#3, AWR scores in VH rats decreased to normal levels. ${ }^{*} P<0.05$, compared with the control group; ${ }^{\#} P<0.05$, compared with the $\mathrm{VH}$ group.
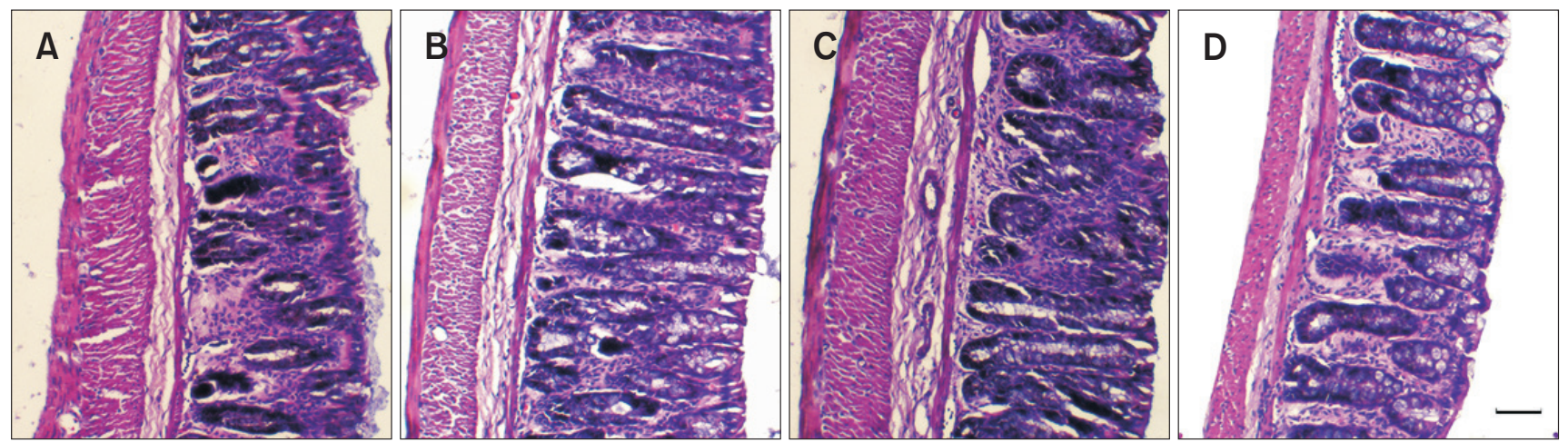

Figure 2. Hematoxylin and eosin staining for colon tissue. (A) Control group, (B) visceral hypersensitivity $(\mathrm{VH})$ group, $(\mathrm{C}) \mathrm{VH}+\mathrm{ketotifen}$ group, and (D) VH + VSL\#3. Scale bar, $200 \mu \mathrm{m}$.
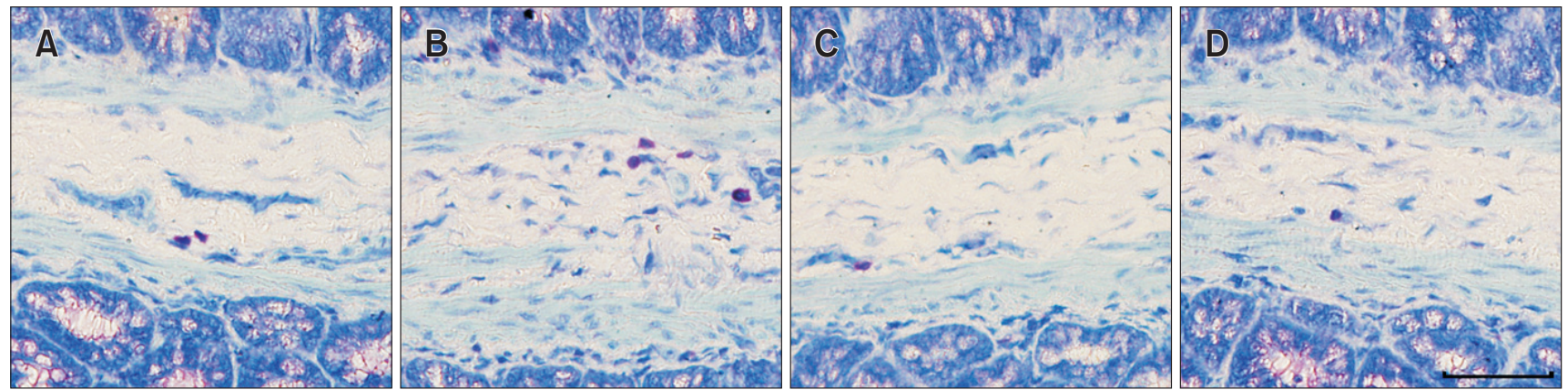

Figure 3. Toluidine blue staining for mast cells. (A) Control group, (B) visceral hypersensitivity (VH) group, (C) VH + ketotifen group, and (D) $\mathrm{VH}+\mathrm{VSL} \# 3$. Scale bar, $50 \mu \mathrm{m}$. 
used after ANOVA analysis where appropriate. Differences with $P$ $<0.05$ were considered to be significant. IBM SPSS version 17.0 statistical software (IBM Corp, Armonk, NY, USA) was used for data processing and analysis.

For microbiota analysis, Wilcoxon test was used in diversity index calculation. Fisher's exact test was performed in cluster analysis. Analysis of species differences between groups was determined by Kruskal-Wallis test. Welch's $t$ test was used in statistical analysis of taxonomic and functional profiles. Differences with $P<0.05$ were considered to be significant.

\section{Results}

\section{Visceral Hypersensitivity}

AWR score was used to evaluate the visceral sensitivity. In the present study, it was revealed that AWR score in response to colorectal distention in the $\mathrm{VH}$ model group $(0.50 \pm 0.40,0.58 \pm$ $0.35,1.16 \pm 0.44$, and $1.33 \pm 0.47)$ was significantly higher than that in the control group $(0.04 \pm 0.11,0.12 \pm 0.17,0.54 \pm 0.47$, and $0.54 \pm 0.31)$. AWR score in the VH + VSL\#3 group $(0.12$ $\pm 0.17,0.29 \pm 0.28,0.54 \pm 0.47$, and $0.88 \pm 0.43)$, or the $\mathrm{VH}$ + ketotifen group $(0.16 \pm 0.17,0.25 \pm 0.24,0.58 \pm 0.50$, and $0.67 \pm 0.44)$ was lower than that in the VH group $(0.50 \pm 0.40$, $0.58 \pm 0.35,1.16 \pm 0.44$, and $1.33 \pm 0.47$ ) (Fig. 1).

\section{Histology of Colonic Tissue}

There was no obvious inflammatory cell infiltration and tissue damage in the colonic mucosa of each group (Fig. 2).

\section{Toluidine Blue Staining for Colonic Mast Cells}

The mast cells were counted under the microscope (Fig. 3). In colonic segments $8 \mathrm{~cm}$ from anus, mast cell number in the $\mathrm{VH}$ group $(4.60 \pm 2.33)$ was significantly higher than that in the control group $(0.17 \pm 0.41)$ (Fig. $4 ; P<0.05)$, and administration of ketotifen $(1.00 \pm 1.73)$ or VSL\#3 $(2.06 \pm 1.47)$ decreased mast cell number in $\mathrm{VH}$ rats (Fig. $4 ; P<0.05$ ). No significant differences were found in colonic segments $3 \mathrm{~cm}$ from anus and ileocecus segments.

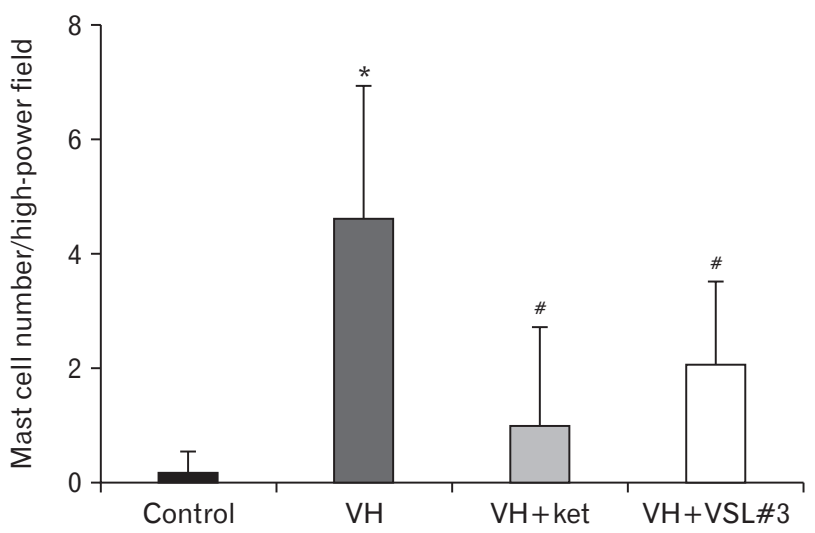

Figure 4. Comparison of mast cell numbers in colonic segments. Mast cell numbers in the visceral hypersensitivity (VH) group were significantly higher than the control group $\left({ }^{*} P<0.05\right)$; ketotifen $($ Ket $)$ and probiotic VSL\#3 decreased mast cell numbers compared with that in the VH group $\left({ }^{\#} \mathrm{P}<0.05\right)$.
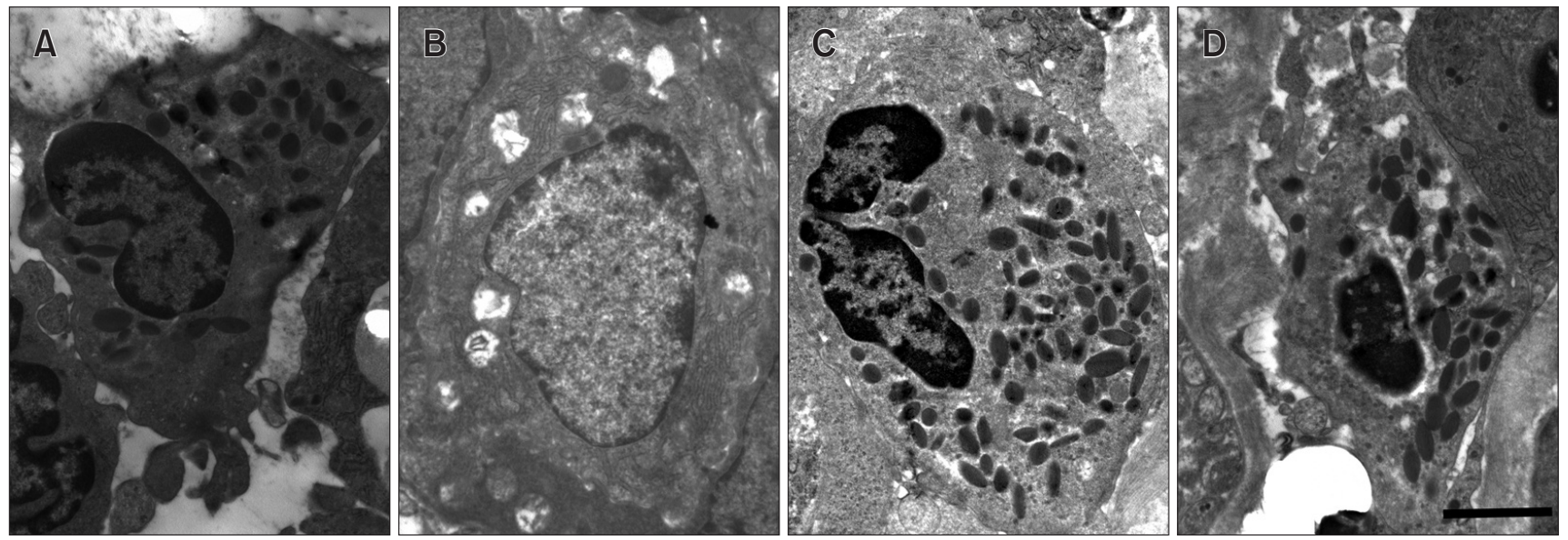

Figure 5. Electron micrograph of mast cell in the rat colon. (A) Control group, (B) visceral hypersensitivity (VH) group, (C) VH + ketotifen group, and (D) VH + VSL\#3. Scale bar, $2 \mu \mathrm{m}$. 


\section{Transmission Electron Microscopic Observation of Colonic Mast Cells}

Mast cells are characterized by the prominent and numerous granules contained in the cytoplasm (Fig. 5). Mast cells are mainly distributed in the lamina propria of intestinal wall. In the $\mathrm{VH}$ group, there are degranulation and vacuole formation, and the degranulation of mast cells is reduced by the intervention of probiotic VSL\#3 or ketotifen (Fig. 5).

\section{Colonic Bacterial Community Structure}

A total of 12 stool samples were analyzed in this study using $16 \mathrm{~S}$ rDNA sequencing. A total of 627321 sequences were finally analyzed and were clustered into 818 OTUs. We compared microbial diversity between the control group and $\mathrm{VH}$ group, $\mathrm{VH}+\mathrm{ke}-$ totifen group and $\mathrm{VH}$ group, and $\mathrm{VH}+\mathrm{VSL} \# 3$ group and $\mathrm{VH}$ group with Coverage, Sobs, Simpson, and Shannon index (Table). No significant differences were found in either 2 groups (Table). Species composition was analyzed (Fig. 6). Firmicutes (68.26\%) and Bacteroidetes $(28.21 \%)$ were the most abundant OTUs in most stool samples (Fig. 6). Actinobacteria and Tenericutes were also detected at very low levels (Fig. 6). The composition of fecal microflora was similar among the control group, $\mathrm{VH}$ group, $\mathrm{VH}$ + ketotifen group, and VH + VSL\#3 group (Fig. 6). We analyzed species with different abundances in the microbial community among groups at the genus level (Fig. 7A). The abundance of Clostridium sensu stricto 1 in the $\mathrm{VH}$ group was higher than that in the control group (Fig. 7B). Treatment with VSL\#3 induced a significant reduction of abundance in Clostridium sensu stricto 1 in $\mathrm{VH}$ rats (Fig. 7C). Clostridium sensu stricto 1 may be associated with VH and may act as a biological target of probiotic VSL\#3 for the treatment of $\mathrm{VH}$.

\section{Discussion}

In the present study, our results showed that probiotic VSL\#3 decreased VH, which had the same effect as the mast cell stabilizer ketotifen. IBS subjects showed the high rate of mast cell degranulation, which increased the release of serotonin, corticotropinreleasing factor and histamine. ${ }^{32}$ Probiotic inhibited $\mathrm{IgE}$-dependent activation and degranulation of mast cell by downregulating highaffinity $\mathrm{IgE}$ receptor 1 and histamine $\mathrm{H} 4$ receptor expression in

Table. Alpha Diversity Indices and Coverage From Gut Bacterial Communities of Rats in Each Group

\begin{tabular}{lccrr}
\hline \multicolumn{1}{c}{ Index } & Control & VH & Ketotifen & VSL\#3 \\
\hline Ace & $0.05 \pm 0.03$ & $0.04 \pm 0.01$ & $0.05 \pm 0.04$ & $0.04 \pm 0.01$ \\
Simpson & $0.10 \pm 0.00$ & $0.10 \pm 0.00$ & $0.10 \pm 0.00$ & $0.10 \pm 0.00$ \\
Shannon & $513.67 \pm 57.50$ & $519.33 \pm 25.93$ & $510.00 \pm 36.00$ & $473.33 \pm 117.25$ \\
Coverage $(\%)$ & $576.28 \pm 51.67$ & $588.71 \pm 19.91$ & $604.66 \pm 22.96$ & $526.28 \pm 108.59$ \\
\hline
\end{tabular}

$\mathrm{VH}$, visceral hypersensitivity.

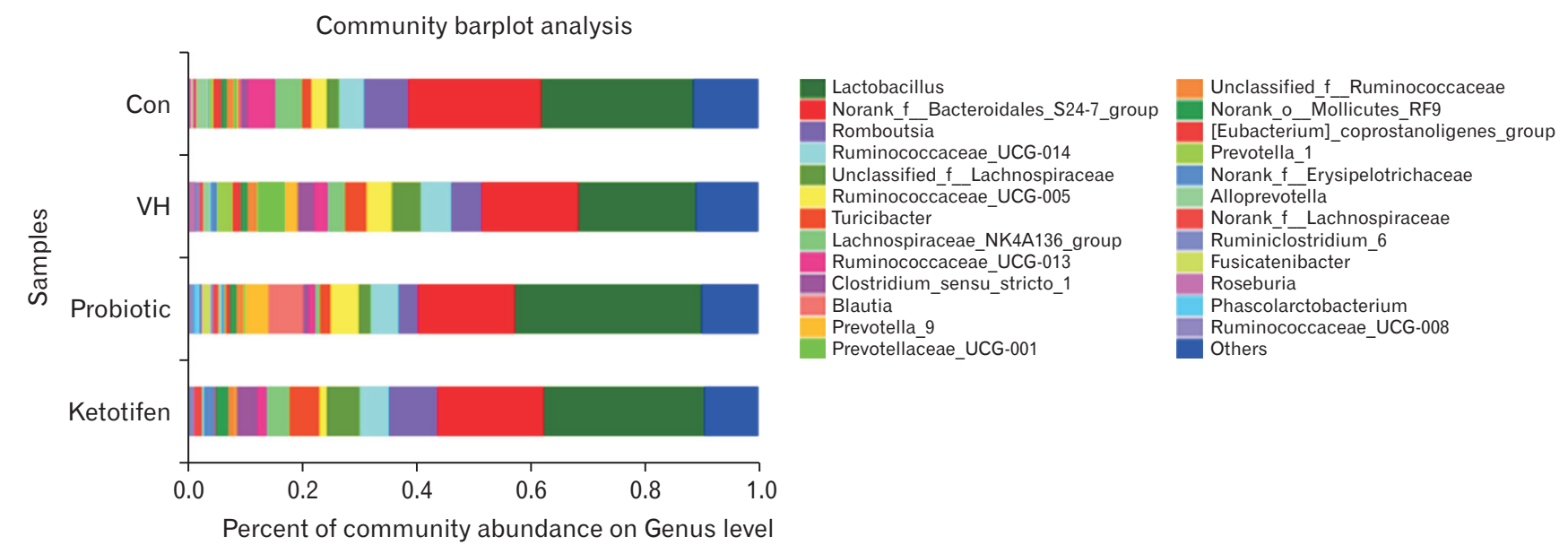

Figure 6. Community bar plot analysis of fecal microbiota in rats. There were no significant differences in bacterial community profiles at the genus level. 

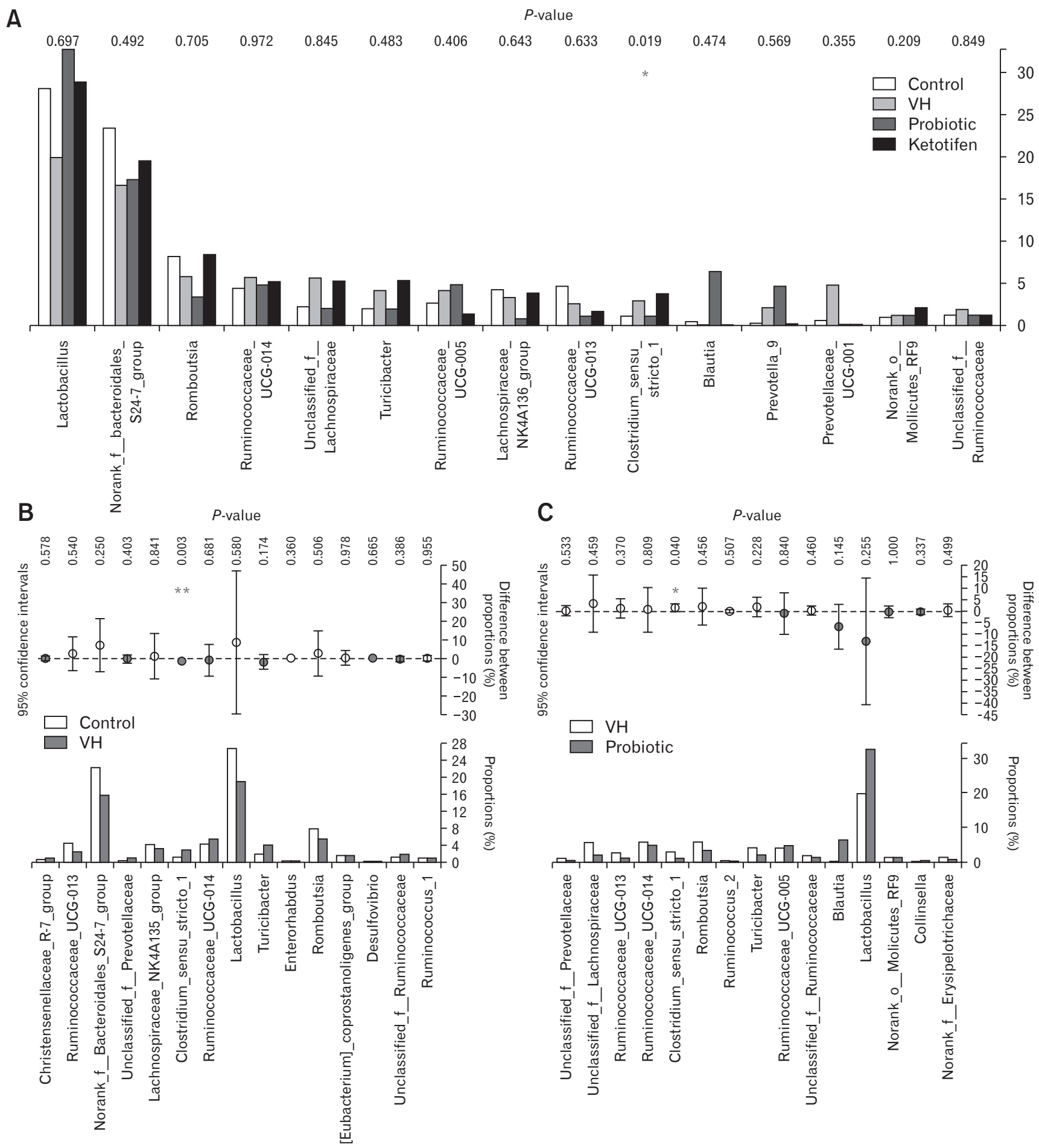

Figure 7. Analysis of species differences at the genus level. (A) Relative abundance of phylotypes in rats. (B) Differences in the relative abundance of phylotypes between visceral hypersensitivity $(\mathrm{VH})$ rats and control rats. $\mathrm{VH}$ rats showed a raised abundance of Clostridium sensu stricto 1 compared with controls. (C) Differences in the relative abundance of phylotypes between $\mathrm{VH}$ rats and VH + VSL\# 3 rats. ${ }^{*} P<0.05 ; 0.001<{ }^{* *} P \leq 0.05$. 
human mast cells. ${ }^{33}$ In addition, probiotic inhibited mast cell degranulation via a Toll-like receptor 2-dependent mechanism with high-affinity IgE receptor 1 gene subunit $\alpha$ downregulation. ${ }^{14,16,17}$ Probiotics such as Bifidobacterium and Bacillus regulated intestinal metabolites by reconstructing intestinal flora structure and affecting intestinal mast-cell immune responses. ${ }^{34,35}$ In this present study, it revealed that both the number and degranulation of mast cells were increased in $\mathrm{VH}$ rats, and the number and degranulation of mast cells could be reduced by the mast cell stabilizer or probiotic VSL\#3. It was further confirmed that mast cells may be related to $\mathrm{VH}$, and probiotic VSL\#3 may reduce $\mathrm{VH}$ via the regulation of mast cell.

Although intestinal microbiota has been linked to IBS, there are only few qualitative data presenting the intestinal microbiota in the experimental models of IBS and IBS patients, and the data are ambiguous. For example, Rajilić-Stojanović et $\mathrm{al}^{36}$ found that there was a significant difference in the composition of intestinal flora (Firmicutes and Bacteroides) between IBS patients and healthy controls, and it was also revealed that the composition of intestinal flora was related to IBS symptoms. Carroll et $\mathrm{al}^{37}$ using $16 \mathrm{~S}$ ribosomal RNA (rRNA) technology to analyze the fecal microbiota of diarrhea-predominant IBS (IBS-D) $(n=23)$ and healthy controls $(n=23)$, it revealed the higher levels of Enterobacteriaceae and the lower levels of Fecalibacterium genera in patients with IBS-D compared to healthy controls. Jeffery et $\mathrm{al}^{38}$ found that compared with healthy controls, IBS-D patients showed normal-like microbiota composition, whereas other IBS subtypes samples $(n=22)$ were characterized by an increase of Firmicutes-associated taxa and a depletion of Bacteroidetes-related taxa. However, Frost et $\mathrm{al}^{26}$ found that there was no difference of fecal microbiota composition between IBS patients and controls. Because of different diagnostic criteria for IBS, ${ }^{39,40}$ the different subtypes of IBS, ${ }^{41}$ the different sampling sites and methods (luminal microflora and intestinal mucosaassociated microflora), ${ }^{42,43}$ the evolution of the bacterial taxonomy, ${ }^{44}$ limitations of methodology for analysis of the microbiota (culturebased methods, ${ }^{41}$ real-time fluorescent quantitative $\mathrm{PCR},{ }^{45}$ and $16 \mathrm{~S}$ $\mathrm{rRNA}^{44}$ ) and microbiota in different geographical environment, ${ }^{46}$ there is no consistent conclusion about the relationship between intestinal microflora and IBS.

Different members of gut bacteria may have different effects on gut function. An increased abundance of Cyanobacteria and Proteobacteria was associated with satiety and bloating, and increased pain threshold. ${ }^{38}$ The increase of Lachnospiraceae and Ruminococcus was correlated with visceral pain. ${ }^{42,47}$ Decrease of Lactobacilli occurred in patients with IBS-D, ${ }^{44}$ constipation-predominant IBS was related to methane excretion. ${ }^{43}$ Lactobacillus plantarum PS128 ameliorated VH through gut-brain axis. ${ }^{48}$ Bifidobacterium longum BB536 and Lactobacillus rhamnosus HN001 restored intestinal permeability and gut microbiota in IBS patients. ${ }^{49}$ Prevotella had a negative association with pain in IBS patients. ${ }^{24}$ Clostridium sensu stricto 1 was a representative species of Clostridium and could produce short-chain fatty acids (SCFAs). ${ }^{50}$ Some studies had demonstrated that there was an increase of Clostridium in stool samples from IBS patients. ${ }^{36,51}$ The relative abundance of Clostridium sensu stricto 1 positively correlated with the content of SCFAs, ${ }^{52}$ which may be correlated with $\mathrm{VH}^{53}$ SUN found that Clostridium increased in IBS-D patients, ${ }^{54}$ further confirming that Clostridium may be associated with IBS. In the present study, we did not find any changes in the composition and diversity of intestinal microbiome between $\mathrm{VH}$ rats and control rats, and the composition and diversity of intestinal microbiome in $\mathrm{VH}$ rats did not change after application of probiotic VSL\#3. However, the fecal microbiota analysis showed that the abundance of Clostridium sensu stricto 1 in $\mathrm{VH}$ rats was higher than that in the normal control group, and the application of probiotic VSL\#3 downregulated the abundance of Clostridium sensu stricto 1 in $\mathrm{VH}$ rats. This result suggested that Clostridium sensu stricto 1 may be related to $\mathrm{VH}$ and a potential mechanism of probiotic VSL\# 3 in the treatment for IBS-D patients.

Overall, in this present study, we found both the number and degranulation of mast cell were increased in $\mathrm{VH}$ rats. The abundance of Clostridium sensu stricto 1 was higher in $\mathrm{VH}$ rats. A mast cell stabilizer and probiotic VSL\#3 could decrease VH, mast cell number and degranulation. Probiotic VSL\#3 simultaneously reduced the abundance of Clostridium sensu stricto 1. Therefore, we suggest that mast cell and intestinal microbiome play a role in the pathogenesis of $\mathrm{VH}$ and probiotic VSL\#3 regulates $\mathrm{VH}$ through mast cell or intestinal microbiome. Due to the small sample in the study, the role of Clostridium sensu stricto 1 in IBS needs further exploration.

In conclusion, the present study revealed that probiotic VSL\#3 was effective in decreasing $\mathrm{VH}$, and further confirmed the role of mast cells in VH rats. The fecal microbiota analysis showed a higher abundance of Clostridium sensu stricto 1 in VH rats, which was reversed by the treatment of probiotic VSL\#3, providing a theoretical foundation for further study on the pathogenesis of IBS. In the latter study, we will further explore the role of mast cells and Clostridium sensu stricto 1 in the pathogenesis of IBS and the mechanism of probiotic VSL\#3 in the treatment of IBS. 
Financial support: This research was supported by National Nature Science Foundation of China (No. 81300273), fund for Scientific Research of The First Hospital of China Medical University (No. FSFH201702), and Liaoning Science and Technology Foundation (No. 20170541052).

\section{Conflicts of interest: None.}

Author contributions: Li Yingjie was responsible for analyzing and interpreting the data, and drafting the manuscript; Jing Li analyzed and interpreted the data; and Cong Dai was corresponding author, and was responsible for designing study, editing, and revising the draft.

\section{References}

1. Sperber AD, Dumitrascu D, Fukudo S, et al. The global prevalence of IBS in adults remains elusive due to the heterogeneity of studies: a rome foundation working team literature review. Gut 2017;66:1075-1082.

2. Drossman DA, Hasler WL. Rome IV-functional GI disorders: disorders of gut-brain interaction. Gastroenterology 2016;150:1257-1261.

3. Larauche MH, Biraud M, Moussaoui N, et al. 65 intracerebroventricular corticotropin-releasing factor (CRF) at low doses induces a CRF1 receptor-mediated visceral analgesia in male rats through recruitment of brain oxytocin. Gastroenterology 2016;150:S16.

4. Bouin M, Plourde V, Boivin M, et al. Rectal distention testing in patients with irritable bowel syndrome: sensitivity, specificity, and predictive values of pain sensory thresholds. Gastroenterology 2002;122:1771-1777.

5. Bednarska O, Walter SA, Casado-Bedmar M, et al. Vasoactive intestinal polypeptide and mast cells regulate increased passage of colonic bacteria in patients with irritable bowel syndrome. Gastroenterology 2017;153:948960, e3.

6. Walker MM, Warwick A, Ung C, Talley NJ. The role of eosinophils and mast cells in intestinal functional disease. Curr Gastroenterol Rep 2011;13:323-330.

7. Klooker TK, Braak B, Koopman KE, et al. The mast cell stabiliser ketotifen decreases visceral hypersensitivity and improves intestinal symptoms in patients with irritable bowel syndrome. Gut 2010;59:1213-1221.

8. Ohashi K, Sato Y, Kawai M, Kurebayashi Y. Abolishment of TNBSinduced visceral hypersensitivity in mast cell deficient rats. Life Sci 2008;82:419-423.

9. Ding SS, Hong SH, Wang C, Guo Y, Wang ZK, Xu Y. Acupuncture modulates the neuro-endocrine-immune network. QJM 2014;107:341345 .

10. Wesolowski J, Paumet F. The impact of bacterial infection on mast cell degranulation. Immunol Res 2011;51:215-226.

11. Xue B, Müller MH, Li J, et al. Mast cells and the cyclooxygenase pathway mediate colonic afferent nerve sensitization in a murine colitis model. Auton Neurosci 2013;174:47-53.

12. Yang CQ, Wei YY, Zhong CJ, Duan LP. Essential role of mast cells in the visceral hyperalgesia induced by T. spiralis infection and stress in rats. Mediators Inflamm 2012;2012:294070.

13. Zhang L, Song J, Hou X. Mast cells and irritable bowel syndrome: from the bench to the bedside. J Neurogastroenterol Motil 2016;22:181-192.

14. Kawahara T. Inhibitory effect of heat-killed Lactobacillus strain on immunoglobulin E-mediated degranulation and late-phase immune reactions of mouse bone marrow-derived mast cells. Anim Sci 2010;81:714721.

15. Kasakura K, Takahashi K, Aizawa T, Hosono A, Kaminogawa S. A TLR2 ligand suppresses allergic inflammatory reactions by acting directly on mast cells. Int Arch Allergy Immunol 2009;150:359-369.

16. Miettinen M, Veckman V, Latvala S, Sareneva T, Matikainen S, Julkunen I. Live Lactobacillus rhamnosus and Streptococcus pyogenes differentially regulate toll-like receptor (TLR) gene expression in human primary macrophages. J Leukoc Biol 2008;84:1092-1100.

17. Takeda K, Kaisho T, Akira S. Toll-like receptors. Annu Rev Immunol 2003;21:335-376.

18. Borre YE, Moloney RD, Clarke G, Dinan TG, Cryan JF. The impact of microbiota on brain and behavior: mechanisms \& therapeutic potential. Adv Exp Med Biol 2014;817:373-403.

19. Schwille-Kiuntke J, Enck P, Zendler C, et al. Postinfectious irritable bowel syndrome: follow-up of a patient cohort of confirmed cases of bacterial infection with Salmonella or Campylobacter. Neurogastroenterol Motil 2011;23:e479-e488.

20. Dupont HL. Review article: evidence for the role of gut microbiota in irritable bowel syndrome and its potential influence on therapeutic targets. Aliment Pharmacol Ther 2014;39:1033-1042.

21. Moloney RD, Desbonnet L, Clarke G, Dinan TG, Cryan JF. The microbiome: stress, health and disease. Mamm Genome 2014;25:49-74.

22. Luczynski P, McVey Neufeld KA, Oriach CS, Clarke G, Dinan TG, Cryan JF. Growing up in a bubble: using germ-free animals to assess the influence of the gut microbiota on brain and behavior. Int J Neuropsychopharmacol 2016;19:pyw020.

23. Aguilera M, Vergara P, Martinez V. Tu2114 visceral pain-related responses are attenuated in mice with antibiotic-induced intestinal dysbiosis. Gastroenterology 2013;144:S-933.

24. Hadizadeh F, Bonfiglio F, Belheouane M, et al. Faecal microbiota composition associates with abdominal pain in the general population. Gut 2018;67:778-779.

25. Dlugosz A, Winckler B, Lundin E, et al. No difference in small bowel microbiota between patients with irritable bowel syndrome and healthy controls. Sci Rep 2015;5:8508.

26. Frost F, Kacprowski T, Rühlemann MC, et al. Functional abdominal pain and discomfort (IBS) is not associated with faecal microbiota composition in the general population. Gut 2019;68:1131-1133.

27. Williams CL, Villar RG, Peterson JM, Burks TF. Stress-induced changes in intestinal transit in the rat: a model for irritable bowel syndrome. Gastroenterology 1988;94:611-621.

28. La JH, Kim TW, Sung TS, Kang JW, Kim HJ, Yang IS. Visceral hypersensitivity and altered colonic motility after subsidence of inflammation in a rat model of colitis. World J Gastroenterol 2003;9:2791-2795.

29. Li YJ, Dai C, Jiang M. Mechanisms of probiotic VSL\#3 in a rat model 
of visceral hypersensitivity involves the mast cell-PAR2-TRPV1 pathway. Dig Dis Sci 2019;64:1182-1192.

30. Dai C, Guandalini S, Zhao DH, Jiang M. Antinociceptive effect of VSL\#3 on visceral hypersensitivity in a rat model of irritable bowel syndrome: a possible action through nitric oxide pathway and enhance barrier function. Mol Cel Biochem 2012;362:43-53.

31. Rijnierse A, Nijkamp FP, Kraneveld AD. Mast cells and nerves tickle in the tummy: implications for inflammatory bowel disease and irritable bowel syndrome. Pharmacol Ther 2007;116:207-235.

32. Zhang L, Song J, Hou X. Mast cell and irritable bowel syndrome: from the bench to the bedside. J Neurogastroenterol Motil 2016;22:181-192.

33. Oksaharju A, Kankainen M, Kekkonen RA, et al. Probiotic Lactobacillus rhamnosus downregulates FCER1 and HRH4 expression in human mast cells. World J Gastroenterol 2011;17:750-759.

34. Żukiewicz-Sobczak W, Wróblewska P, Adamczuk P, Silny W. Probiotic lactic acid bacteria and their potential in the prevention and treatment of allergic diseases. Cent Eur J Immunol 2014;1:104-108.

35. Walsh CJ, Guinane CM, O’Toole PW, Cotter PD. Beneficial modulation of the gut microbiota. FEBS Lett 2014;588:4120-4130.

36. Rajilić-Stojanović M, Biagi E, Heilig HG, et al. Global and deep molecular analysis of microbiota signatures in fecal samples from patients with irritable bowel syndrome. Gastroenterology 2011;141:1792-1801.

37. Carroll IM, Ringel-Kulka T, Siddle JP, Ringel Y. Alterations in composition and diversity of the intestinal microbiota in patients with diarrheapredominant irritable bowel syndrome. Neurogastroenterol Motil 2012;24:521-530, e248.

38. Jeffery IB, O’Toole PW, Öhman L, et al. An irritable bowel syndrome subtype defined by species-specific alterations in faecal microbiota. Gut 2012;61:997-1006.

39. Drossman DA, Hasler WL. Rome IV-functional GI disorders: disorders of gut-brain interaction. Gastroenterology 2016;150:1257-1261.

40. Malinen E, Rinttilä T, Kajander K, et al. Analysis of the fecal microbiota of irritable bowel syndrome patients and healthy controls with real-time PCR. AM J Gastroenterol 2005;100:373-382.

41. Taverniti V, Guglielmetti S. Methodological issues in the study of intestinal microbiota in irritable bowel syndrome. World J Gastroenterol 2014;20:8821-8836.

42. Esquerre N, Basso L, Defaye M, et al. Colitis-induced microbial perturbation promotes post-inflammatory visceral hypersensitivity. Cell Mol
Gastroenterol Hepatol 2020;10:225-244.

43. Pimentel M, Chow EJ, Lin HC. Normalization of lactulose breath testing correlates with symptom improvement in irritable bowel syndrome. a double-blind, randomized, placebo-controlled study. Am J Gastroenterol 2003;98:412-419.

44. Lo Presti A, Del Chierico F, Altomare A, et al. Exploring the genetic diversity of the 16S rRNA gene of Akkermansia muciniphila in IBD and IBS. Future Microbiol 2019;14:1497-1509.

45. Rinttilä T, Lyra A, Krogius-Kurikka L, Palva A. Real-time PCR analysis of enteric pathogens from fecal samples of irritable bowel syndrome subjects. Gut Pathog 2011;3:6.

46. Wu GD, Chen J, Hoffmann C, et al. Linking long-term dietary patterns with gut microbial enterotypes. Science 2011;334:105-108.

47. Labus JS, Osadchiy V, Hsiao EY, et al. Evidence for an association of gut microbial Clostridia with brain functional connectivity and gastrointestinal sensorimotor function in patients with irritable bowel syndrome, based on tripartite network analysis. Microbiome 2019;7:45.

48. Liu YW, Wang YP, Yen HF, et al. Lactobacillus plantarum PS128 ameliorated visceral hypersensitivity in rats through the gut-brain axis. Probiotics Antimicrob Proteins 2020;12:980-993.

49. Bonfrate L, Di Palo DM, Celano G, et al. Effects of Bifidobacterium longum BB536 and Lactobacillus rhamnosus HN001 in IBS patients. Eur J Clin Invest 2020;50:e13201.

50. Gupta RS, Gao B. Phylogenomic analyses of clostridia and identification of novel protein signatures that are specific to the genus Clostridium sensu stricto (cluster I). Int J Syst Evol Microbiol 2009;59(Pt 2):285-294.

51. Distrutti E, Monaldi L, Ricci P, Fiorucci S. Gut microbiota role in irritable bowel syndrome: new therapeutic strategies. World J Gastroenterol 2016;22:2219-2241.

52. Yan H, Lu J, Wang Y, Gu W, Yang X, Yu J. Intake of total saponins and polysaccharides from polygonatum kingianum affects the gut microbiota in diabetic rats. Phytomedicine 2017;26:45-54.

53. Kamath PS, Hoepfner MT, Phillips SF. Short-chain fatty acids stimulate motility of the canine ileum. Am J Physiol 1987;253(4 Pt 1):G427-G433.

54. Sun YY, Li M, Li YY, et al. The effect of Clostridium butyricum on symptoms and fecal microbiota in diarrhea-dominant irritable bowel syndrome: a randomized, double-blind, placebo-controlled trial. Sci Rep 2018;8:2964. 